\title{
Compact, Low-power and Precision Timing Photodetector Readout
}

\author{
Gary S. Varner* \\ Dept. of Physics and Astronomy, University of Hawaii \\ E-mail:varner@phys.hawaii.edu

\section{Larry L. Ruckman} \\ Dept. of Physics and Astronomy, University of Hawaii
}

\section{Jochen Schwiening, Jaroslav Va'vra}

Stanford Linear Accelerator Center

\begin{abstract}
Photodetector readout for next generation high event rate particle identification and single-photon detection requires a digitizer capable of integrated recording of dense arrays of sensor elements with high analog bandwidth (precision timing) and large record depth, in a cost-effective, compact and low-power way. Simply stated, one cannot do better than having a high-fidelity "oscilloscope on a chip" for every sensor channel. A first version of the Buffered Large Analog Bandwidth (BLAB1) ASIC has been designed based upon the lessons learned from the development of the Large Analog Bandwidth Recorder and Digitizer with Ordered Readout (LABRADOR) ASIC. While this LABRADOR ASIC has been very successful and forms the readout basis of a generation of new, large-scale radio neutrino detectors, its limited sampling depth is a major drawback. To address this shortcoming, a prototype intended for photodetector readout has been designed and fabricated with $64 \mathrm{k}$ deep sampling at multi-GSa/s operation. An evaluation system has been constructed for instrumentation of Time-Of-Propagation (TOP) and focusing DIRC prototypes and test results will be reported.
\end{abstract}

International workshop on new photon-detectors PD07

June 27-29 2007

Kobe University, Kobe, Japan

\footnotetext{
${ }^{*}$ Speaker.
} 


\section{Introduction}

Many future applications of advanced photodetectors require a readout system capable of recording large numbers of channels operating at high signal rates with precise amplitude and temporal resolution. A traditional technique for single photon timing recording uses a Constant Fraction Discrimination (CFD) followed by fine resolution Time-to-Digital Conversion (TDC). For precision timing applications, a measure of amplitude may also be required for residual thresholdamplitude corrections. In addition, rate dependent (or pile-up) corrections may be necessary.

Specific examples of configurations being considered for a Super B-Factory Particle Identification detector may be seen in Fig. 1. Due to poorer $K / \pi$ separation capability at higher particle momentum, the Bar Time-Of-Propagation (TOP) detector [1] - with a modest number of channels - has been abandoned. The remaining viable Čerenkov detector options, either a focusing (TOP) [2] or a focusing Detector of Internally Reflected Čerenkov (fDIRC) [3, 4, 5] require potentially large numbers of readout channels, ranging from many 10's to 100's of thousands of instrumented channels. The space available for the photodetectors inside either an upgraded BaBar or Belle detector is very limited. Therefore the photodetectors, readout electronics and cabling must be very space efficient.

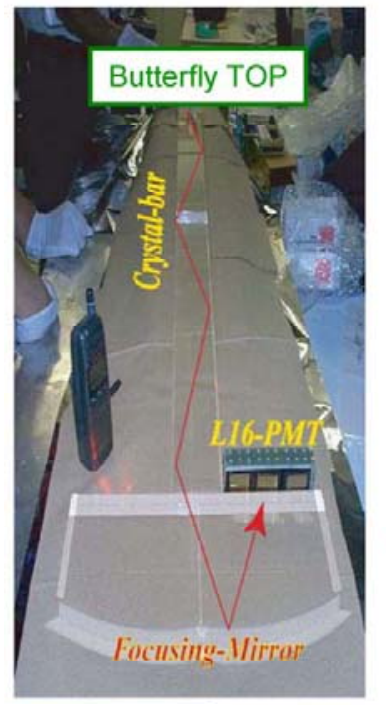

$\sim 1 \mathrm{~mm}$ pos. resolution: $200 \mathrm{Ch} /$ counter $* 180$ counters $=36,000$ channels

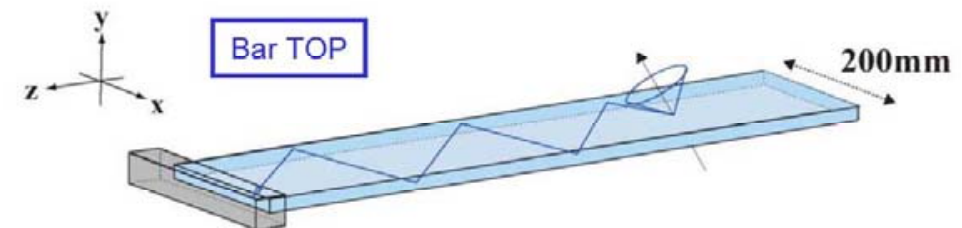

Photon detector $\quad \sim 5 \mathrm{~mm}$ pos, resolution: $40 \mathrm{Ch} /$ counter

* 200 counters $=1440$ channels

Multi-hit (hidden cost) $>1440$ channels

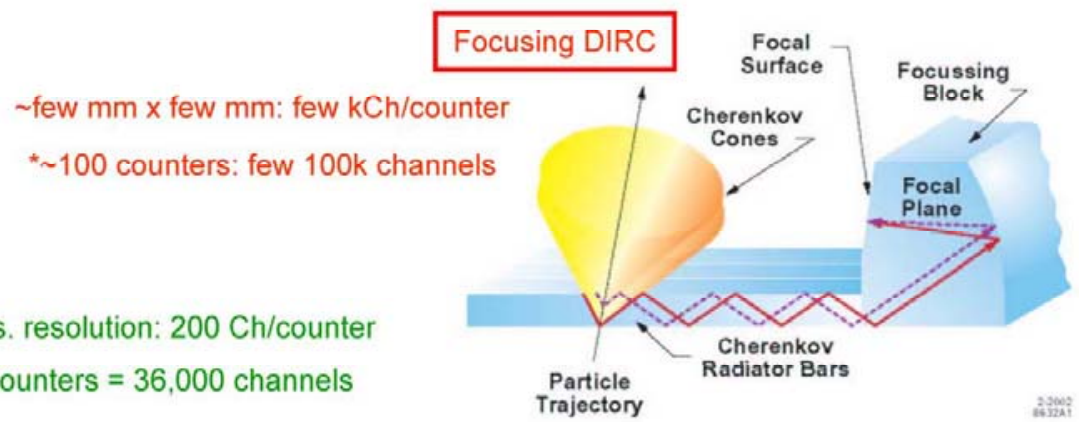

Figure 1: Particle identification technologies considered for Super B-Factory upgrade.

We present a compact readout technique that is capable of operating at very high rates while requiring lower power, with an emphasis toward high integration with the photodetectors. To do so inside a detector with large numbers of channels it is desirable to remove potentially large noise due to the significant number of fast switching discriminators. 


\section{High Speed Waveform Sampling}

If affordable, the most general method of reading out any detector channel is via a very high analog bandwidth, high sampling speed digital sampling oscilloscope. With sufficient signal recording fidelity, a wide variety of signal feature extraction/zero suppression algorithms may subsequently be used. These techniques remain viable even in the presence of severe background and pile-up. Dense configurations of inexpensive Switched Capacitor Array (SCA) devices have been developed, for instance [6] [7, 8]. These devices have already found applications with existing detectors $[9,10,11]$ and hold promise for the future [12], where the readout of large focal plane arrays of Multi-Pixel Photon Counters (MPPCs) strongly benefit from such inexpensive, high performance recording.

For precision time recording applications, while these devices have multi-GSa/s sampling rates, an improvement of analog bandwidth is required. At the same time, for radio detection of Ultra High Energy neutrinos by the Antarctic Impulsive Transient Antenna (ANITA) [13] and Askaryan Underice Radio Array (AURA) [14], an analog bandwidth on the order of $1 \mathrm{GHz}$ is needed. These requirements on high analog bandwidth in addition to high sampling speed led to the evolution [15] of the Large Analog Bandwidth Recorder And Digitizer with Ordered Readout (LABRADOR) ASIC [16]. Fig. 2 left shows a die photograph of a LABRADOR ASIC, a die size of approximately 10 square mm. At right is a detailed schematic of this 9-channel chip. Analog input values are available on a common input bus line. Great effort was made to make the input coupling and this microstrip bus line $50 \Omega$ impedance. A write pointer, common to all 9 channels, is generated by a ripple oscillator chain whose intermediate stages are XORed to form individual timing gate signals that open and close the storage capacitor cells. Upon external detection of a trigger condition, a hold signal is issued and sampling is halted (ripple oscillator continues but write pointer logic is stopped). To speed conversion and readout, each storage cell contains a dedicated Wilkinson ADC. That is, a common voltage ramp signal is applied to a comparator in each cell and a common Gray code count is latched upon crossing threshold for the stored voltage value. This allows conversion to 12 bits of resolution in as little as $31 \mu \mathrm{s}$, while transferring the 2340 digitized samples at $50 \mathrm{MHz}$ requires just under $50 \mu \mathrm{s}$.

The performance of this device is comparable to a commercial digital sampling oscilloscope as seen at the left of Fig. 3. However, both the power and cost of this "oscilloscope on a chip" are at least two orders of magnitude smaller.

On the right side of the figure is plotted the distribution of the bin-by-bin widths of each of almost 19,000 storage cells flown on the ANITA 06-07 Antarctic campaign. Calibration was performed using a $600 \mathrm{MHz}$ reference clock. Monte Carlo estimates indicate that after this correction sub 30ps timing resolution between radio pulses should be possible for infinite Signal-to-Noise Ratios (SNR). For realistic SNRs, 50ps or better timing resolution has been obtained.

\section{Deeper Buffer Depth}

This successful device led to the desire to develop a deep storage (Buffered) version of the LABRADOR ASIC [17] (denoted BLAB1), targeted toward large array and collider detector applications, which require much longer record lengths. The die photograph of such a device is 

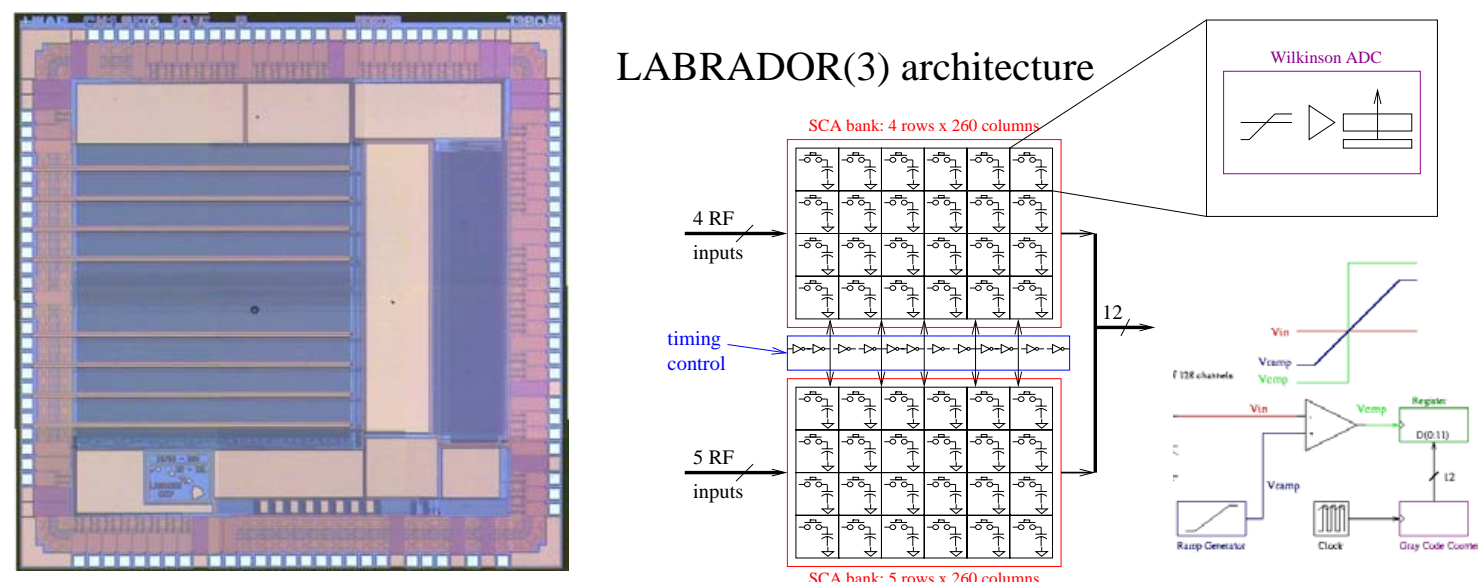

Figure 2: LABRADOR ASIC die photo (left) and functional schematic (right), where the die is about $10 \mathrm{~mm}^{2}$ and all storage samples may be converted in parallel, as used in the ANITA and AURA detectors. Digitization to 12-bits and readout can be performed in less than $100 \mu \mathrm{s}$.
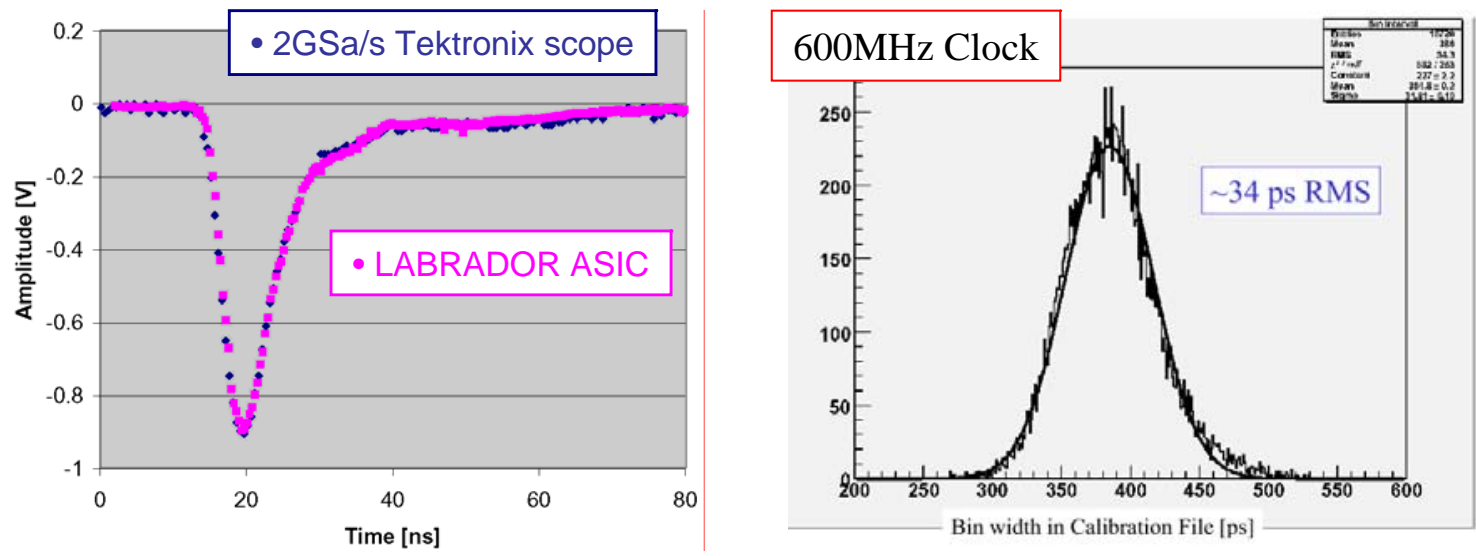

Figure 3: At left, LABRADOR ASIC analog comparison versus commercial oscilloscope, where the comparison is for a cosmic ray muon signal in a Belle Time-Of-Flight counter with both a commercial oscilloscope (blue points) and LABRADOR (magenta points). At right is the right for bin-by-bin timing calibration performance of the more than 18,700 storage cells flown on the ANITA payload.

displayed on the left side of Fig. 4, which is only slightly larger than the LABRADOR die shown earlier. However, instead of a $2.4 \mathrm{k}$ storage cells, this device has $64 \mathrm{k}$ deep sampling on a single channel. In order to obtain this density, clock and register components of the Wilkinson conversion were moved to a companion FPGA. The noise results are histogrammed in the center plot, where noise comparable to the LABRADOR is obtained. Notably, in the rightmost plot, the new sample pointer logic is faster, allowing sampling in excess of $5 \mathrm{GSa} / \mathrm{s}$.

\section{Precision Photodetector Readout}

High timing precision, multi-channel single photon detectors, such as Micro-Channel Plate 

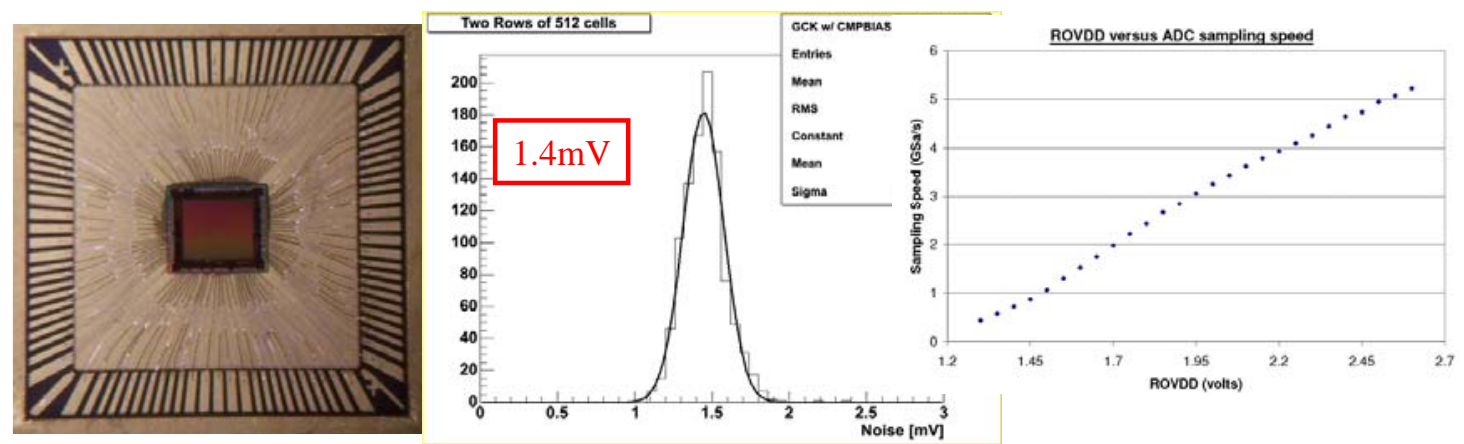

Figure 4: Buffered LABRADOR (BLAB1) ASIC die photo (left) and functional schematic (right), where the die is about $10 \mathrm{~mm}^{2}$ and all storage samples may be converted in parallel.

(MCP) PMTs, typically have relatively low gain compared with that needed for use with standard discriminators and waveform sampling devices. To address this limitation, experience from radio detection work [19] led to the use of inexpensive, RF amplifiers in this readout prototype. A diagram illustrating the signal flow and board hardware for realizing amplification and BLAB1 readout of a 64-channel MCP PMT is found in Fig. 5. At bottom left is a stack of four 16-channel amplifier cards. This compact array of cards fits within the footprint of an $8 \times 8$ array element MCP PMT. Each card consists of inexpensive amplifiers, though some care is needed in layout to prevent oscillations with the large analog bandwidths provided.

Output signals are differential analog and transmitted via a power module (not shown) to a digitizer CAMAC module, shown at lower right. This module consists of 16-channels of BLAB1 waveform recording and a large FPGA for data processing. For triggered events, feature extracted information (leading edge time, peak amplitude) is collected via CAMAC, while entire waveform information is logged via USB2.0 to a nearby data collection PC.

\section{High Precision Timing}

With the full waveform sampling, one is capable of implementing elaborate signal feature timing extraction. An example of this capability is given at the left of Fig. 6, where the ability to measure the period of a $400 \mathrm{MHz}$ sine wave is compared against zero crossing determinations from the sampled waveforms. No bin-by-bin correction is applied, only an overall slew dependence across the chip (due to supply voltage drop).

It is seen that roughly an $11 \mathrm{ps}$ RMS timing residual is obtained, including the tail. The core of the distribution is approximately 8ps and is the goal for improved calibration. At right is the timing jitter on the measured period between two injected square pulses, nominally 30ns apart. The residual is $27 \mathrm{ps}$, indicating something like 20ps per edge timing determination. For a more realistic input signal (PMT curvature), the timing resolution degrades to something like 40ps for a linear leading edge fit about the 50\% point of the waveform. More sophisticated algorithms are being explored to improve this performance. 


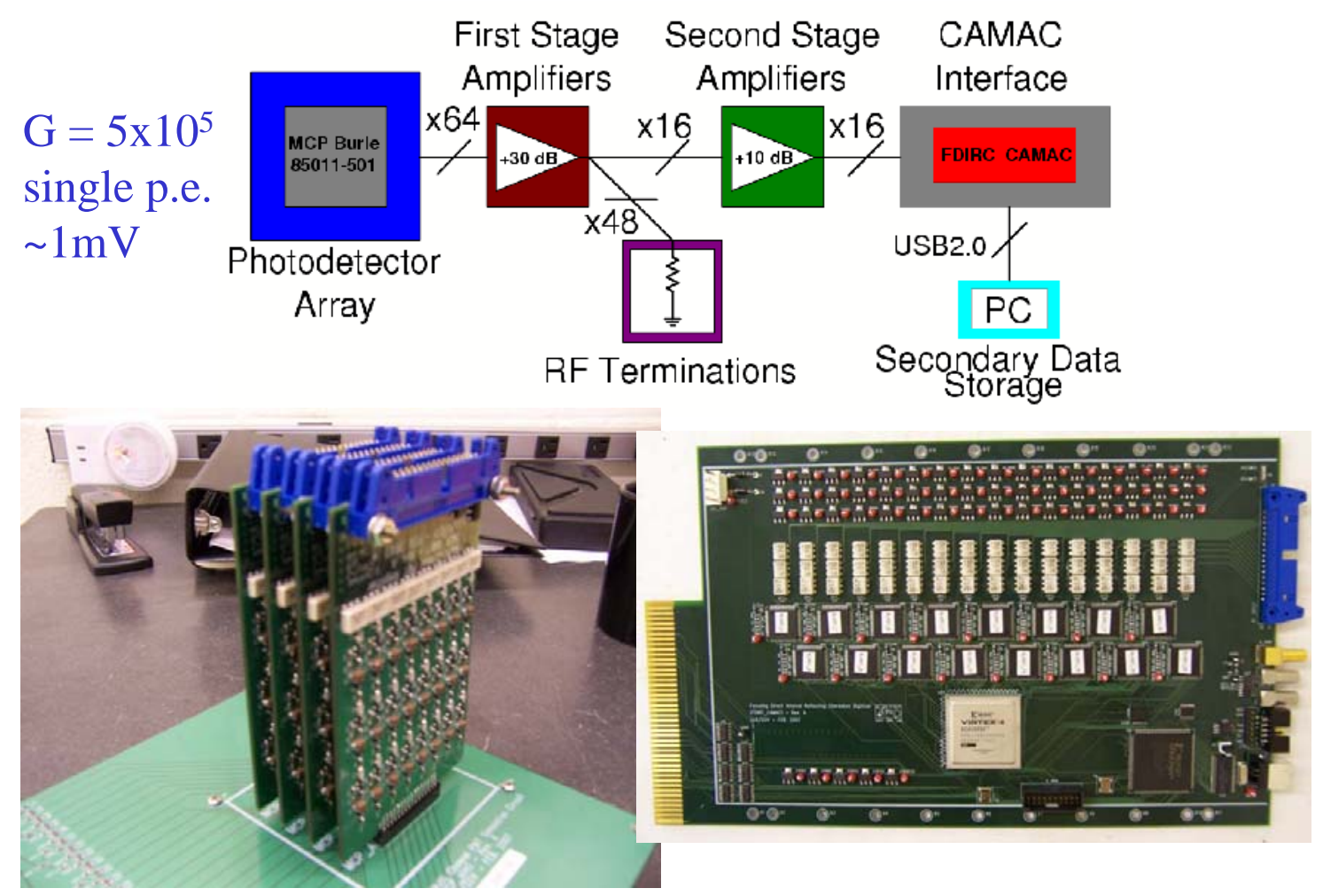

Figure 5: Signal flow through the prototype readout. At the top is a schematic flow, at lower left the stack of amplifier cards, and at right is a 16-channel digitizer card, implemented for convenience with the rest of the data acquisition as a CAMAC module. To permit more detailed waveform analysis, full waveforms are read out from this CAMAC card via USB2.0 connector on the front panel to a PC.

\section{Focusing DIRC Prototype Tests}

In order to explore the performance of a focusing DIRC, a detector prototype has been constructed at SLAC, a schematic diagram of which is shown at left of Fig. 7. This detector has been studied extensively with GEANT4, as indicated at the right of this figure. Readout of this prototype consists of custom CFDs, developed at SLAC, and commercial ADC and TDC modules.

A further test beam run, designated T-492, will be performed in August 2007. It will include at least 16 and possible 32 channels of the prototype readout described above. Results will be reported later. In preparation for these tests, single photon waveforms have been recorded in the "scanning setup" at SLAC, displays of which are seen in Fig. 8. Notable in the right side of this figure is the cross-talk seen on the signal channels, a feature of the prototype Burle MCP-PMTs utilized. Burle engineers are aware of the issue and plan to remediate via better base layout in future tubes. Tests of the electronics alone indicate that cross-talk intrinsic to the electronics is less than $1 \%$. Careful inspection of this ringing indicates that it should be possible to mitigate such effects by carefully determining cross-coupling and taking advantage of the temporal latency of this crosstalk. 

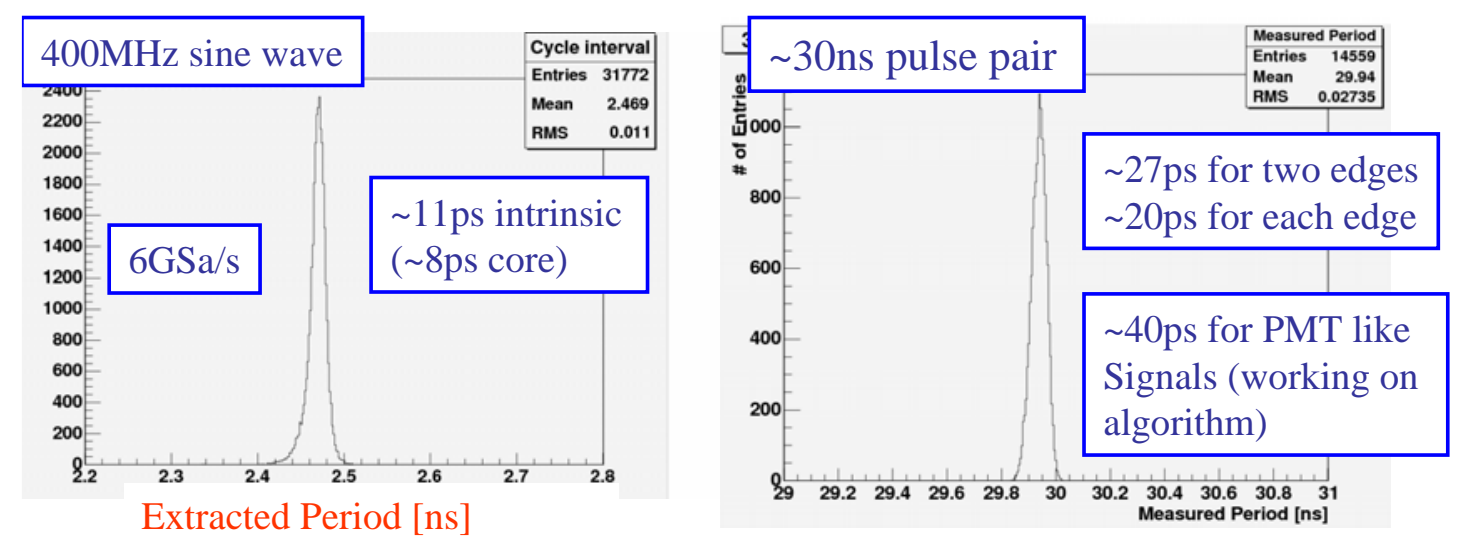

Figure 6: Examples of precision timing recording using the instrumentation array. At left is the timing residual on fitted versus actual period for a $400 \mathrm{MHz}$ sine wave signal. At right is the jitter on the measured time interval between pulses 30ns apart, where a linear fit has been done to the $50 \%$ points of the injected signals. It is noted that for PMT-like signals, with leading edge curvature, the comparable timing resolution degrades to 40ps for a linear leading-edge fit.
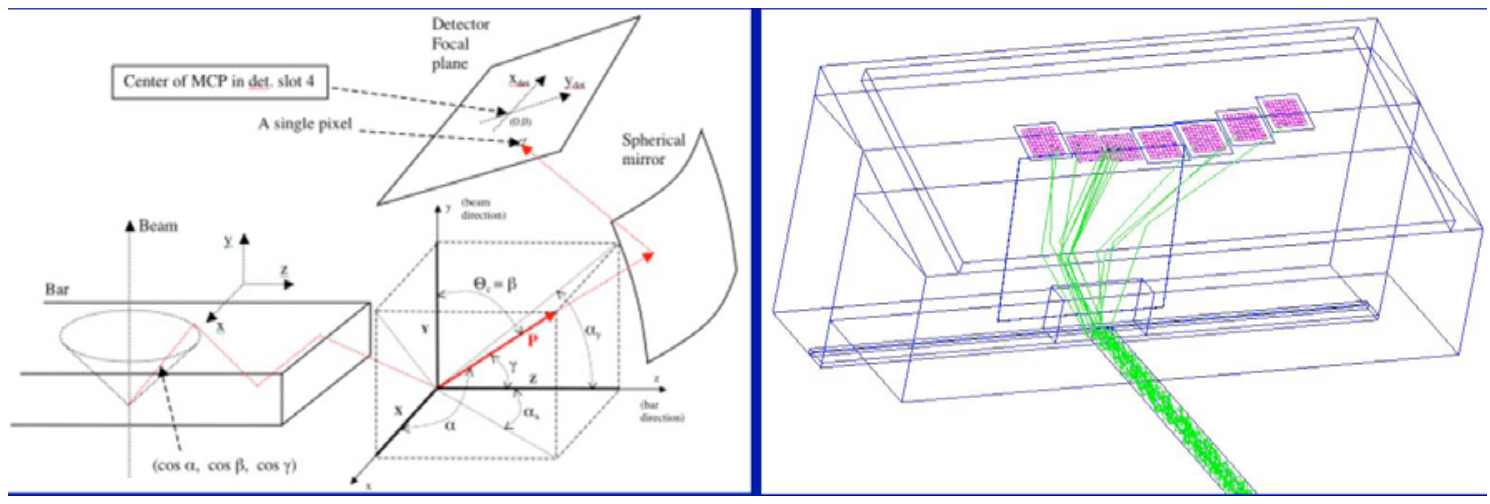

Figure 7: At left is a schematic diagram of the Čerenkov emission pattern being imaged onto the focal plane array of the T-492 focusing DIRC prototype. At the right is a GEANT4 simulation of an electron image onto the photodetector array. The instrumentation described records these single photon hits with high timing precision.

\section{Summary}

A prototype system for precision time recording of single photon signals in a high-rate environment has been presented. Bench test results indicate that time resolutions commensurate with the transit-time spreads of the best multi-channel, single photon detectors are capable. This system will be characterized with test beam as part of the T-492 experiment at SLAC. This technique shows great promise for low-cost and high-performance readout of large photodetector arrays requiring excellent temporal resolution. 

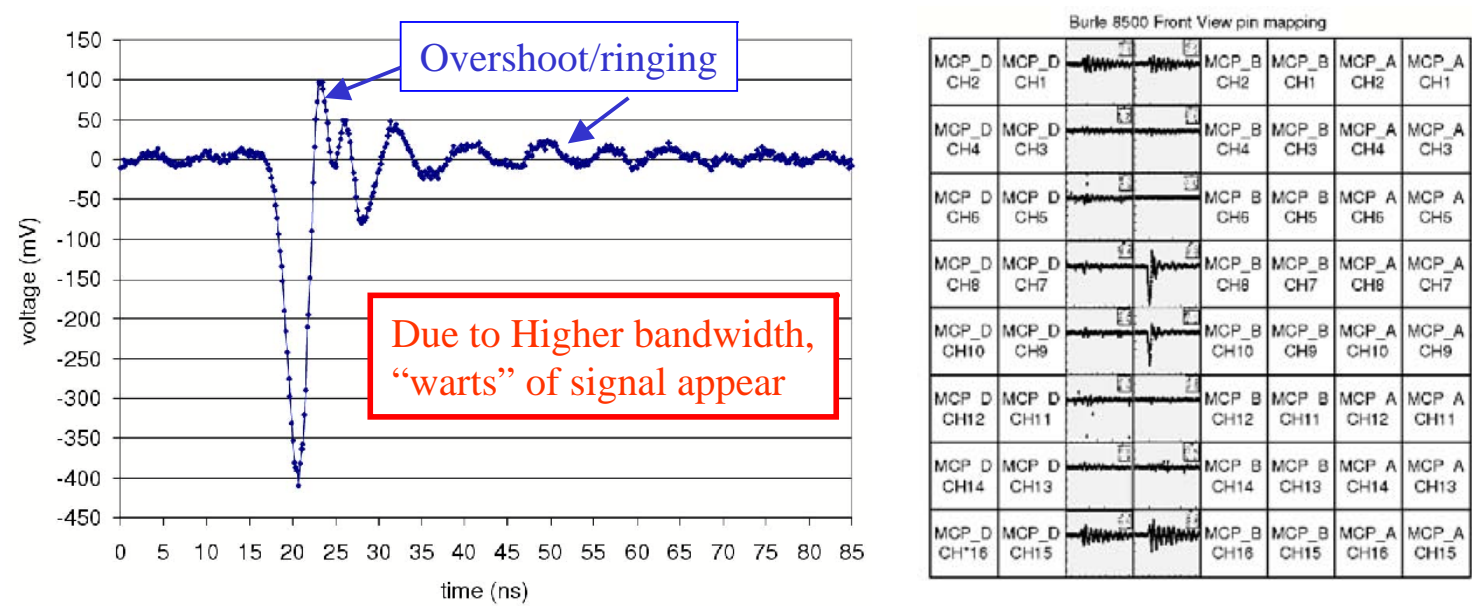

Figure 8: Example recording of a single photon waveform in the SLAC MCP PMT scanning set-up. At left is the waveform in the channel illuminated, at right is illustrated the cross-talk observed on the prototype Burle PMT used in the T-492 test set-up, a feature seen previously though more sharply refined with higher bandwidth. Burle is aware of this issue and plans to remediate by better grounding in future MCP-PMTs.

\section{Acknowledgements}

This work was supported by generous support from the Department of Energy in general and in particular by the Advanced Detector Research program.

\section{References}

[1] A. Akatsu et al., Nucl. Instr. Meth. A440 (1999) 124.

[2] Y. Mazuka, "Aerogel RICH and TOP Counter for Super KEKB," Presented at the 3rd SuperB Workshop at SLAC, econf: C0606141/talks/DAY1/108.PDF June 2006.

[3] T. Kamae et al., Nucl. Instr. Meth. A382 (1996).

[4] J. Benitez et al., "Development of a Focusing DIRC", Prepared for 2006 IEEE Nuclear Science Symposium, SLAC-PUB-12236.

[5] J. Va'vra, "Focusing DIRC R\&D", Presented at the 3rd SuperB Workshop at SLAC, econf: C0606141/talks/DAY1/109.PDF June 2006.

[6] S. Kleinfelder, IEEE Trans. Nucl. Sci. 50 (2003) 955.

[7] C. Brönnimann, R. Horisberger and R. Schnyder, Nucl. Instr. Meth. A420 (1999) 264.

[8] S. Ritt, Nucl. Instr. Meth. A518 (2004) 470

[9] F. Suekane et al. (KamLAND Collaboration), Prog. Part. Nucl. Phys. 57 (2006) 106.

[10] R. Stokstad et al. (IceCube Collaboration), "Design and Performance of the IceCube Electronics," Presented at the 11th Workshop on Electronics for LHC and Future Experiments, 12-16 Deptember 2005, Heidelberg, Germany.

[11] E. Delagnes et al., Nucl. Instr. Meth. A567 (2006) 21. 
[12] E. Lorentz, these proceedings.

[13] P. Gorham et al., (ANITA Collaboration), Submitted to Phys. Rev. Lett., hep-ex/0611008, SLAC-PUB-12286.

[14] H. Landsman, A. Karle et al. (IceCube Collaboration) and G. Varner and L. Ruckman, to be published International Cosmic Ray Conference 2007 proceedings.

[15] G.S. Varner et al., Monolithic Multi-channel GSa/s Transient Waveform Recorder for Measuring Radio Emissions from High Energy Particle Cascades, Proc. SPIE Int.Soc.Opt.Eng. 4858 (2003) 31.

[16] G. Varner et al., "The Large Analog Bandwidth Recorder And Digitizer with Ordered Readout (LABRADOR) ASIC," arXiv:physics/0509023v2, submitted to NIM A.

[17] G. Varner et al., J. Instr. 1 (2006) P07001.

[18] L. Ruckman and G. Varner, Photodetector Readout Monolithic with Precision Timing, to appear Proceedings of the 2007 IEEE Nuclear Science Symposium.

[19] G.S. Varner et al. (ANITA Collaboration), Detection of Ultra High Energy Neutrinos via Coherent Radio Emission, Proceedings of International Symposium on Detector Development for Particle, Astroparticle and Synchrotron Radiation Experiments (SNIC 2006) pp 0046, SLAC-PUB-11872. 